Research Paper

\title{
Effects of Hypobaric Hypoxia on Rat Retina and Protective Response of Resveratrol to the Stress
}

\author{
Xiaorong Xin ${ }^{\bowtie}$, Hong Dang, Xiaojing Zhao, Haohao Wang \\ Department of Ophthalmology, Qinghai Red Cross Hospital, Xining, Qinghai, China \\ $\triangle$ Corresponding author: Xiaorong Xin, M.D. Department of Ophthalmology, Qinghai Red Cross Hospital, Xining 810000, Qinghai Province, China. Email: \\ xrgc19@yahoo.com \\ (C) Ivyspring International Publisher. This is an open access article distributed under the terms of the Creative Commons Attribution (CC BY-NC) license \\ (https://creativecommons.org/licenses/by-nc/4.0/). See http://ivyspring.com/terms for full terms and conditions.
}

Received: 2017.01.29; Accepted: 2017.06.28; Published: 2017.08.17

\begin{abstract}
High-altitude retinopathy represents retinal functional changes associated with environmental challenges imposed by hypobaric hypoxia, but the detailed cellular and molecular mechanism underlying this process remains unclear. Our current investigation was to explore the effect of hypobaric hypoxia on the rat retina and determine whether resveratrol has a protective efficacy on the hypoxic damage to the retina. Experiment rats were randomly grouped as the control group, hypoxia group and resveratrol intervention group. The hypoxia group and the resveratrol intervention group were maintained in a low-pressure oxygen cabin, and the resveratrol intervention group was given daily intraperitoneal injections with resveratrol. We found that hypobaric hypoxia increased thioredoxin 1 (Trxl) and thioredoxin 2 (Trx2) expression in retinas, and resveratrol treatment significantly reversed these changes $(P<0.05, P<0.05$ respectively). In comparison with controls, hypoxia upregulated the mRNA expression levels of caspase3 $(P<0.001)$, caspase9 $(P<0.01)$, heat shock protein $70(\mathrm{Hsp70})(P<0.05)$, heat shock protein $90(\mathrm{Hsp} 90)(P<0.001)$ and hypoxia-inducible factor-1 (HIF-1) $(P<0.05)$. Resveratrol administration caused a significant decrease in the gene expression of caspase3 $(P<0.001)$, HSP90 $(P<0.05)$ and HIF-1 mRNA $(P<0.01)$ as well as an increase in HSP70 mRNA when compared with the hypoxia group. These findings indicated that resveratrol exerted an anti-oxidative role by modulating hypoxia stress- associated genes and an anti-apoptosis role by regulating apoptosis-related cytokines. In conclusion, hypobaric hypoxia may have a pathological impact on rat retinas. The intervention of resveratrol reverses the effect induced by hypobaric hypoxia and elicits a protective response to the stress.
\end{abstract}

Key words: Hypobaric hypoxia, Retina, Resveratrol, Caspase, Heat shock protein, Hypoxia-inducible factor-1.

\section{Introduction}

Increasing numbers of people are traveling to high altitudes, where the inadequacy of oxygen for the body's metabolic requirements initiates a series of physiologic changes such as increased cardiac output and ventilation. Travelers ascending above $3000 \mathrm{~m}$ exposed to low barometric pressure are therefore susceptible to numerous acute altitude illnesses such as acute mountain sickness, high-altitude cerebral edema, and high-altitude pulmonary edema [1-3]. High-altitude retinopathy, first described in 1969 [4], represents retinal vascular decompensation associated with hypoxia. Studies regarding the acute mountain sickness revealed that an ascent to high altitude might cause morphological and functional changes of the retina, such as optic disk swelling, vitreous hemorrhage, retinal and choroidal blood flow alteration, and retinal hemorrhages $[5,6]$, but the detailed cellular and molecular mechanism underlying this pathological process remains unclear.

Resveratrol, a polyphenol phytoalexin belonging to stilbene (3,4',5-trihydroxystilbene) class [7], has been found in various plants and fruits, and is especially abundant in grapes and red wine [8]. Emerging evidence has demonstrated that protective roles of resveratrol might be involved in pathological conditions including anti-inflammation, antioxidation, antiangiogenesis, anti-carcinogenesis, and anti-apoptosis [9-12]. Resveratrol has been considered to exert a fundamental role in neuroprotection in multiple neurodegenerative diseases such as Alzheimer's, Parkinson's, and Huntington's disease [13]. Neuroprotective effects of 
resveratrol include elimination of reactive oxygen species (ROS), reduction of DNA fragmentation, and enhancement of the regulatory effects on growth factors and anti-inflammatory cytokines [14]. Considering that retina is an extension of the central nervous system, we postulated that resveratrol might be involved in the retinal protection against hypobaric hypoxia stress. The present study was designed to investigate the molecular mechanisms by which hypobaric hypoxia induces retinal impairment, and to explore the effects of resveratrol administered as a preventive agent in hypoxic retinal injury in rats.

\section{Materials and Methods}

\section{Animals}

All animal experiments were approved by the ethical committee of Qinghai Medical College (Qinghai, China). Animal care and experimental procedures were conducted in accordance with the recommendations in the Guide for the Care and Use of Laboratory Animals of the National Institutes of Health. Male Sprague-Dawley rats (SPF-class; weight:190 - $230 \mathrm{~g}$, purchased from Experimental Animal Center of Xian Communication University, Certificate number: SCXK Shanxi 2012-003) were maintained in a clean animal room with a temperature of $22{ }^{\circ} \mathrm{C}-25^{\circ} \mathrm{C}$ and relative humidity of approximately $55 \%$ under a $12 \mathrm{~h}$ light/dark cycle. Rats were housed in the animal room for 2 weeks to adapt to the environment before hypoxic experiment.

\section{Experimental models}

After adaptive feeding for two weeks, 48 SD rats were randomly divided into three groups: control group, hypoxia group and resveratrol intervention group. The hypoxia group and the resveratrol intervention group were maintained in a low-pressure oxygen cabin (simulation for $5000 \mathrm{~m}$ above sea level). The resveratrol intervention group was given daily intraperitoneal injections with resveratrol (30 mg/ $\mathrm{kg}$, Biotopped, China), and the hypoxia group was administered intraperitoneally with the same dose of normal saline at the same time.

\section{Immunohistochemistry staining}

All animals were euthanized after 7 days of treatment, and fresh retina tissues obtained from each group were fixed immediately in $4 \%$ formalin solution, dehydrated by grade alcohol, and embedded in paraffin.

Sections were deparaffinized and rehydrated followed by permeabilization with $0.05 \%$ Triton X-100. The tissue was blocked with 3\% bovine serum albumin and incubated with primary antibodies against thioredoxin-interacting protein (Txnip) (1:150), thioredoxin 1 (Trx1) (1:100), and thioredoxin 2 (Trx2) (1:100) (Proteintech, USA) overnight at $4^{\circ} \mathrm{C}$ and then washed with PBST. Anti rabbit secondary antibody (1:500) (Jackson ImmunoResearch, USA) was applied and incubated for $30 \mathrm{~min}$ at $37^{\circ} \mathrm{C}$. The antigen was finally visualized by peroxidase staining with the peroxidase substrate 3, 3'-diaminobenzidine tetrahydrochloride. Sections were counterstained with hematoxylin, dehydrated and mounted. Semi-quantitative immunostaining analysis was evaluated in retina tissue sections.

\section{Real-time reverse transcription-polymerase chain reaction (real-time $R T-P C R$ ) analysis}

For real-time RT-PCR analysis, total RNA was extracted from the rat retina using RNAiso Plus reagent (TaKaRa, Shiga, Japan). Approximately $0.5 \mu \mathrm{g}$ of the extracted RNA was reverse-transcribed into cDNA using PrimeScript RT Master Mix (TaKaRa, Shiga, Japan). Real-time PCR was performed with a SYBR Green PCR Master Mix (ABI, USA) according to the manufacturer's protocol. The cycling conditions are as follows: $95^{\circ} \mathrm{C}$ for $3 \mathrm{~min}, 40$ cycles of $95^{\circ} \mathrm{C}$ for $10 \mathrm{~s}$, and $60^{\circ} \mathrm{C}$ for $30 \mathrm{~s}$. Relative gene expression was normalized by the median expression of $\beta$-actin as a housekeeping gene. The primer sets used in this study are listed in Table 1.

\section{Statistical analysis}

Statistical analyses were performed by SPSS v18.0 software (SPSS Inc., Chicago, IL), and the data were presented as mean \pm standard deviation (SD). One way Analysis of Variance followed by post hoc analysis was used to identify the differences among groups. Statistical significance was defined as $P<0.05$.

Table 1. List of primers used for quantitative real-time RT-PCR

\begin{tabular}{lll}
\hline Primer & Forward $\left(5^{\prime}-3^{\prime}\right)$ & Reverse $\left(5^{\prime}-3^{\prime}\right)$ \\
\hline HIF-1 & GCTGCCTCTTCGACAAGCTT & CGCTGGAGCTAGCAGAGTCA \\
caspase3 & GGA CCT GTG GAC CTG AAA & GGG TGC GGT AGA GTA AGC \\
caspase9 & GCC TCA TCATCA ACA ACG & CTG GTA TGG GAC AGC ATCT \\
Hsp70 & GGCTAGAGACAGACTCTTGATGG & CTCAGTTTGTAGGGATGCAAGG \\
Hsp90 & GGTCATCTTGCTGTACGAAA & GGTGGCATTTCTTCAGTTAC \\
$\beta$-actin & CCCATCTATGAGGGTTACGC & TTTAATGTCACGCGATTTC \\
\hline
\end{tabular}

HIF-1: hypoxia-inducible factor-1; Hsp70: heat shock protein 70; Hsp90: heat shock protein 90 


\section{Results}

\section{Immunostaining of Txnip}

Positive expression of Txnip was observed in all the three groups (Fig. 1A, Fig. 1B, and Fig. 1C). Moderate positive expression was seen in the inner plexiform layer and nerve fiber layer, and mild expression was found in the outer plexiform layer. As shown in Fig. 1D, Txnip expression increased in hypoxia condition compared to the control group. Resveratrol administration resulted in a decrease in Txnip expression, when compared to the hypoxia group. Overall, no statistical differences were found among the three groups.

\section{Immunostaining of Trx I}

More obvious Txr1 expression was found in the inner plexiform layer and nerve fiber layer in the hypoxia group (Fig. 2B) compared to controls (Fig. 2A). In resveratrol intervention group (Fig. 2C), Txr1 expression in the nerve fiber layer and inner plexiform layer was weaker than those in hypoxia group. Mild expression was observed in the outer plexiform layer in all conditions. As shown in Fig. 2D, hypoxia induced an increase in Trx1 expression compared to the control group $(P<0.01)$. Resveratrol intervention markedly downregulated Trx1 expression in retinas as compared to untreated hypoxic samples $(P<0.05)$.

\section{Immunostaining of Trx2}

In control retinas, Trx2 moderate positive immunostaining was observed in the inner plexiform layer and nerve fiber layer. Mild expression was showed in the outer plexiform layer (Fig. 3A). More obvious positive expression was found in the inner plexiform layer, and mild expression was exhibited in the outer plexifrom layer under hypoxic condition (Fig. 3B). In resveratrol intervention group, mild expression was seen in the nerve fiber layer and inner plexiform layer (Fig. 3C). As demonstrated in Fig. 3D, exposure to hypoxic environment enhanced the Trx2 expression in retinas compared to controls. By contrast, resveratrol treatment reversed these changes and decreased $\operatorname{Tr} x 2$ expression in the retina, compared with those in the hypoxia group $(P<0.05)$.

\section{Resveratrol administration suppressed the hypoxia-induced upregulation of HIF-1 MRNA}

To investigate the involvement of HIF-1 regulation in hypobaric hypoxic stress, we examined the HIF-1 mRNA expression by real-time PCR analysis. Our findings revealed that the relative HIF-1 mRNA expression level was significantly increased in the hypoxia group compared to controls $(P<0.05)$. HIF-1 mRNA expression was notably decreased in the resveratrol intervention group $(P<0.01)$, when compared to the hypoxia group, indicating that resveratrol administration attenuated the hypoxia-induced upregulation of HIF-1mRNA (Fig. 4).
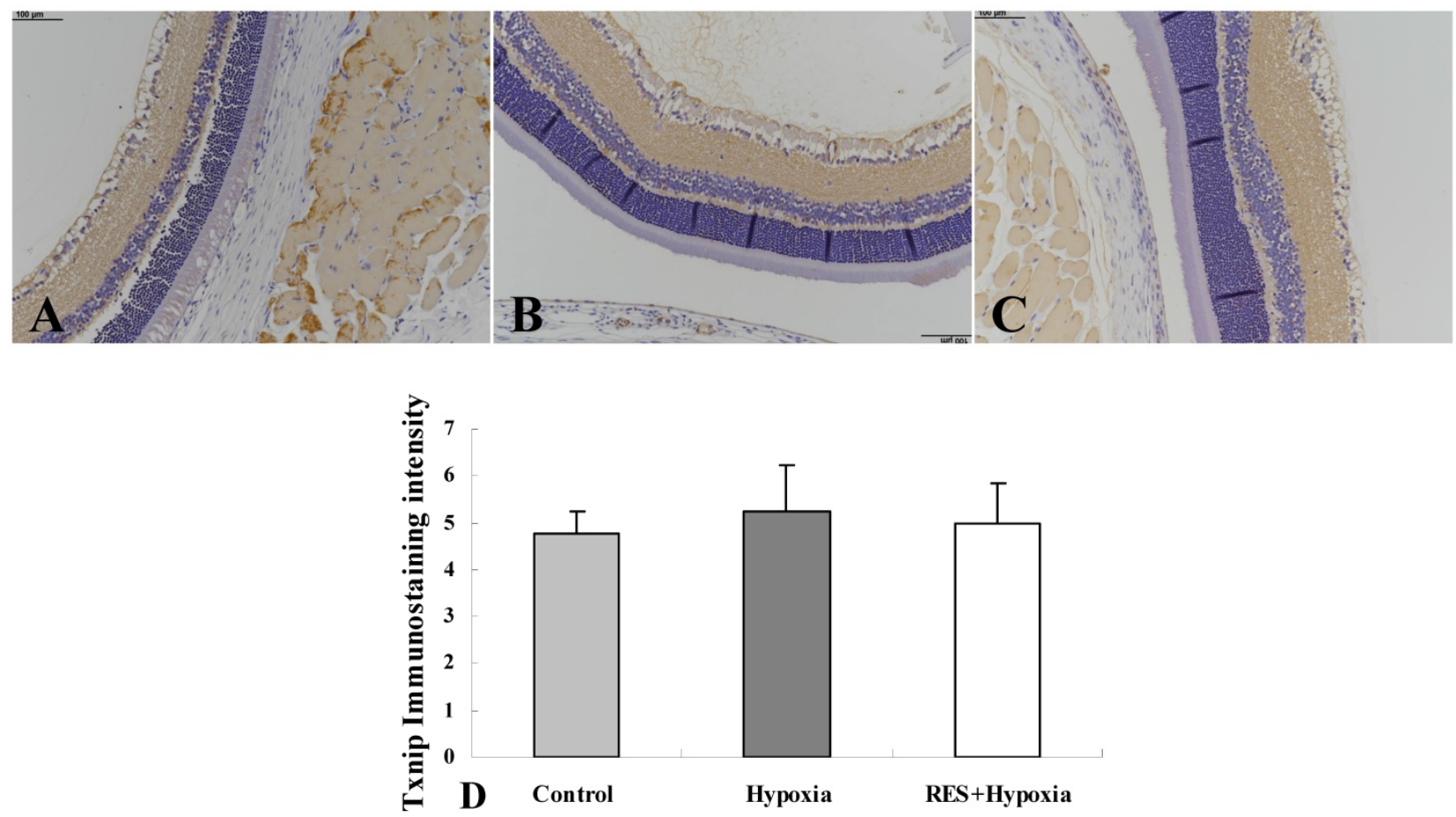

Figure 1. Immunostaining of Txnip expression in rat retinas. (A) Control group; (B) Hypoxia group; (C) Resveratrol intervention (RES + Hypoxia) group; (D) Positive immunostaining intensity of Txnip expression. Results are presented as mean \pm standard deviation (SD) $(n=4)$. Scale bar $=100 \mu \mathrm{m}$. 

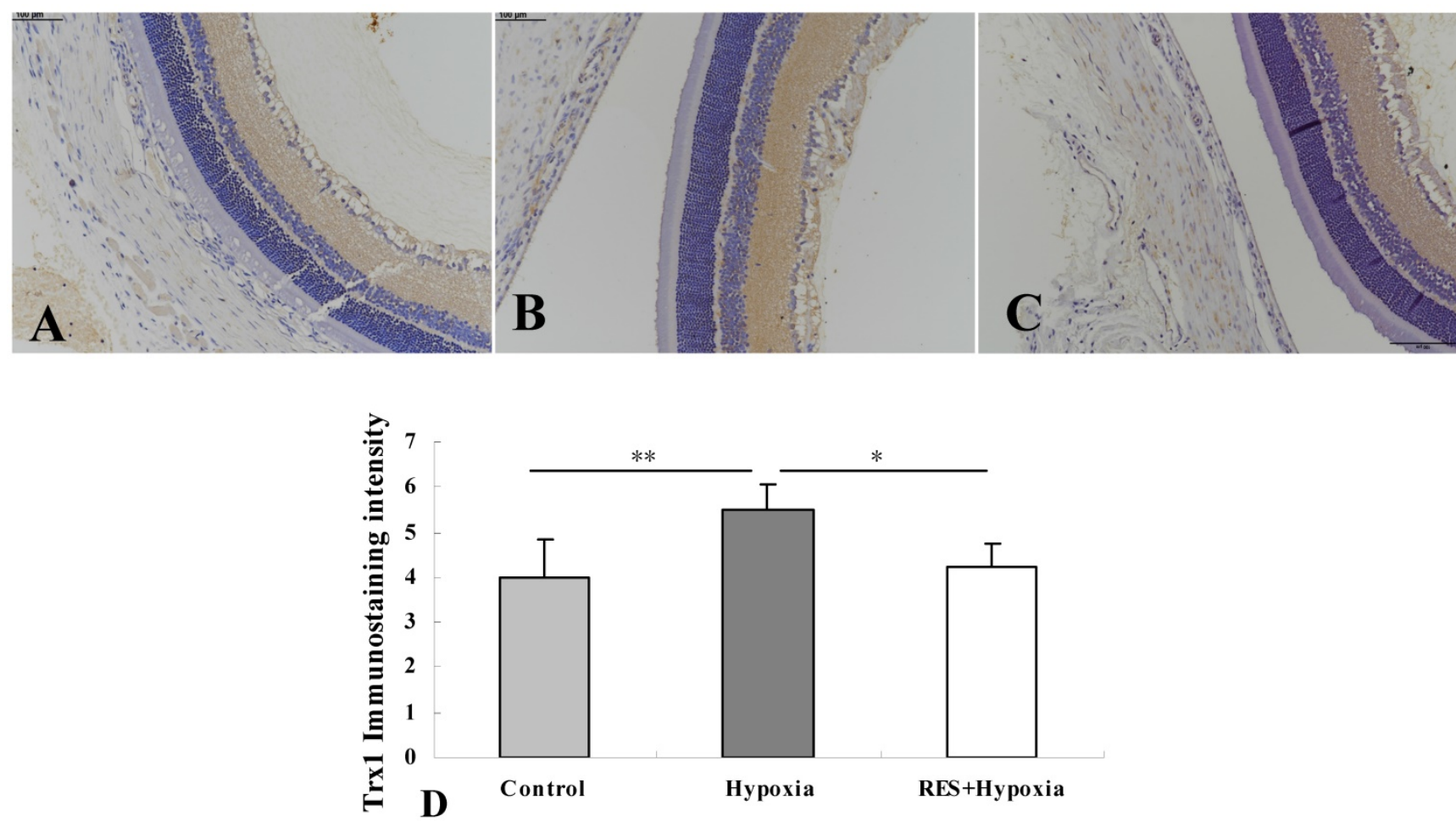

Figure 2. Immunohistochemistry micrographs of Trxl expression in rat retinas. (A) Control group; (B) Hypoxia group; (C) Resveratrol intervention (RES + Hypoxia) group; (D) Positive immunostaining intensity of Trxl expression. Results are presented as mean \pm standard deviation (SD) $(n=4) .{ }^{*} P<0.05 ;{ }^{* *} P<0.01$. Scale bar $=100 \mu \mathrm{m}$.
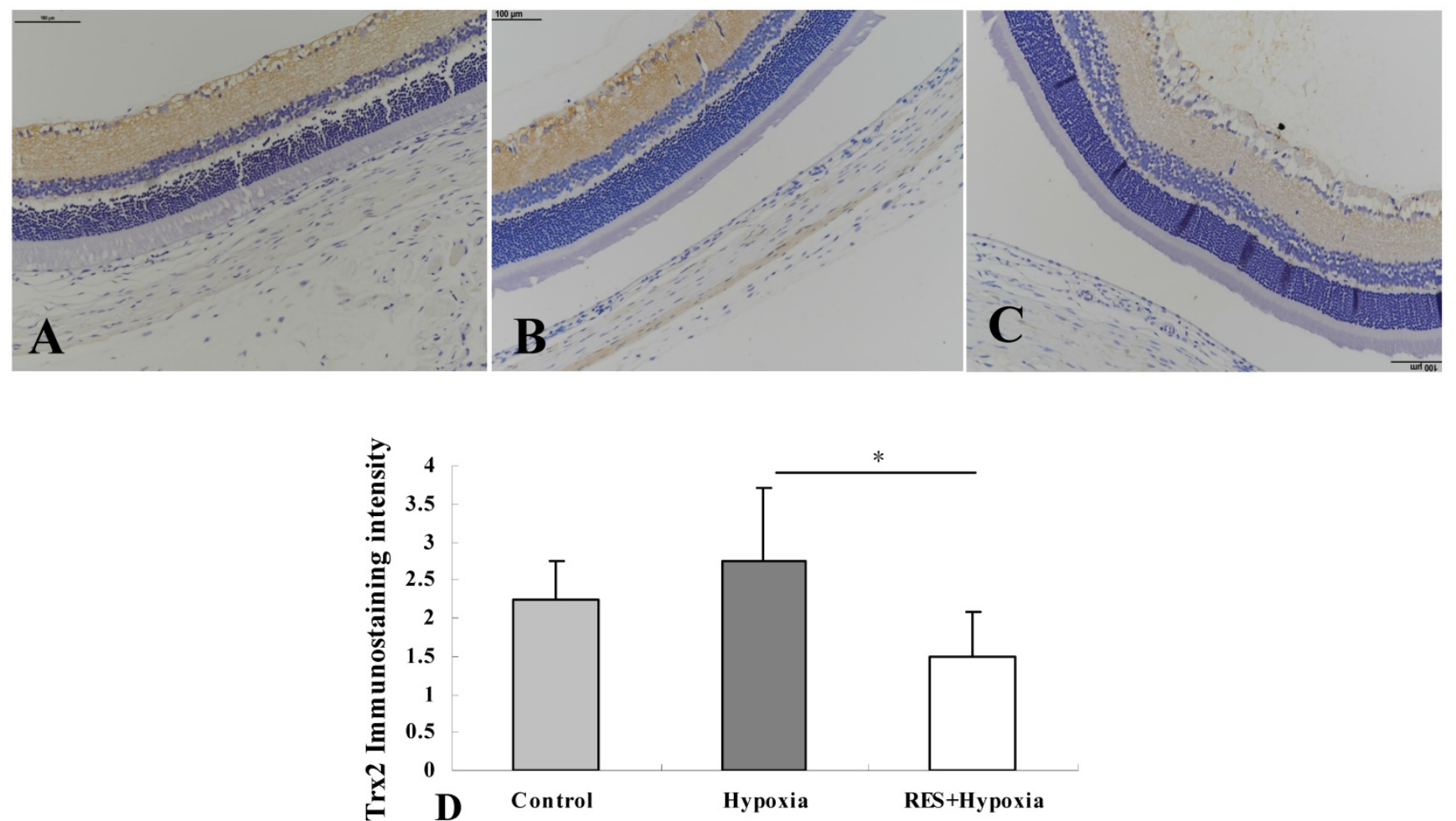

Figure 3. Immunohistochemical images of Trx2 expression in rat retinas. (A) Control group; (B) Hypoxia group; (C) Resveratrol intervention (RES + Hypoxia) group; (D) Positive immunostaining intensity of Trx2 expression. Results are presented as mean \pm standard deviation $(S D)(n=4)$. ${ }^{*} P<0.05$. Scale bar $=$ $100 \mu \mathrm{m}$. 


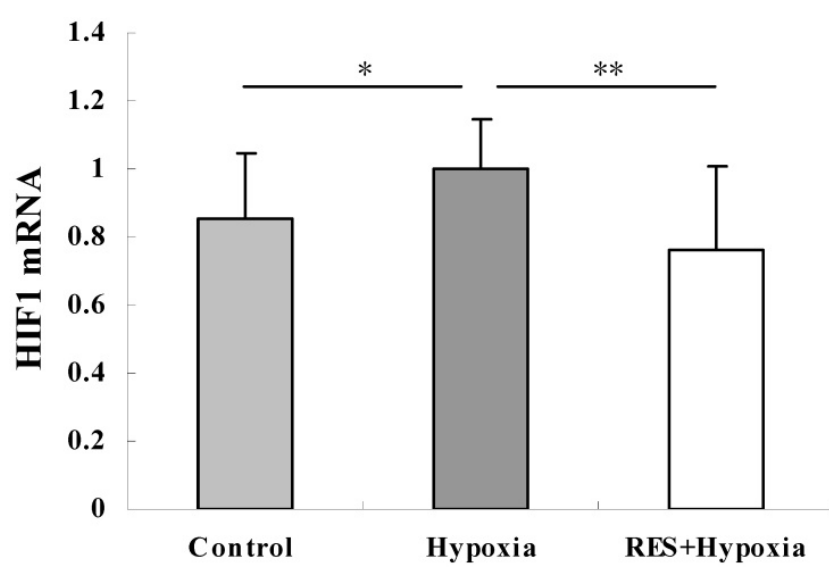

Figure 4. HIF-1 mRNA expression in retinas in control, hypoxia and resveratrol invention (RES + Hypoxia) groups. Results are presented as mean \pm standard deviation (SD) $(n=6)$. All experiments were performed at least in triplicate. $* P<0.05, * * p<0.01$.

\section{Resveratrol treatment attenuated the hypoxia-induced increase in caspase 9 and caspase 3 expression in the retina}

Major mechanisms leading to apoptosis consist of the extrinsic pathway and the intrinsic pathways mediated by mitochondrial damage. To verify whether apoptosis was involved in hypobaric hypoxia-induced stress, we assessed mRNA levels of caspase3 and caspase9, which are mitochondria-dependent apoptosis related factors. Compared to the control group, the mRNA expression of caspase9 was increased under hypoxia condition $(P<0.01)$. Reduction of caspase9 mRNA expression in the resveratrol intervention group was observed as compared to the hypoxia group, but there was no significant difference $(P>0.05)$ (Fig. 5A). Under hypoxic stress, caspase3 mRNA expression was significantly increased relative to those in controls $(P<0.001)$. These results suggested that hypobaric hypoxia could induce apoptosis via mediating caspase3 and caspase9 activity. In comparison with hypoxia group, resveratrol treatment markedly decreased the hypoxia-induced upregulation of caspase 3 mRNA $(P<0.001)$ (Fig. 5B). This result revealed that resveratrol alleviated hypobaric hypoxia-induced apoptosis in retinas.

\section{Resveratrol supplementation enhanced the level of Hsp70 mRNA and inhibited the hypoxia-induced upregulation of Hsp90 mRNA}

Analysis of Hsp70 expression was performed to confirm the effects of hypoxia on the mRNA level of the gene. Compared to controls, the mRNA expressions of Hsp70 was elevated in the hypoxia group $(P<0.05)$, resveratrol intervention further enhanced Hsp70 expression $(P<0.01)$ (Fig. 6A). Hsp70 overexpression can inhibit events occurring downstream of caspase activation [15]. Our result implied that resveratrol administration played an anti-apoptosis role through upregulating Hsp70.

We assessed Hsp90 mRNA level to determine the effect of resveratrol administration on Hsp90 expression. As demonstrated in Fig. 6B, significant upregulation of Hsp90 was detected under hypoxic condition compared to controls $(P<0.001)$. Reduction of Hsp90 mRNA expression in the resveratrol intervention group was observed when compared to the hypoxia group, indicating that resveratrol treatment significantly inhibited the hypoxia-induced upregulation of Hsp90 mRNA $(P<0.05)$.
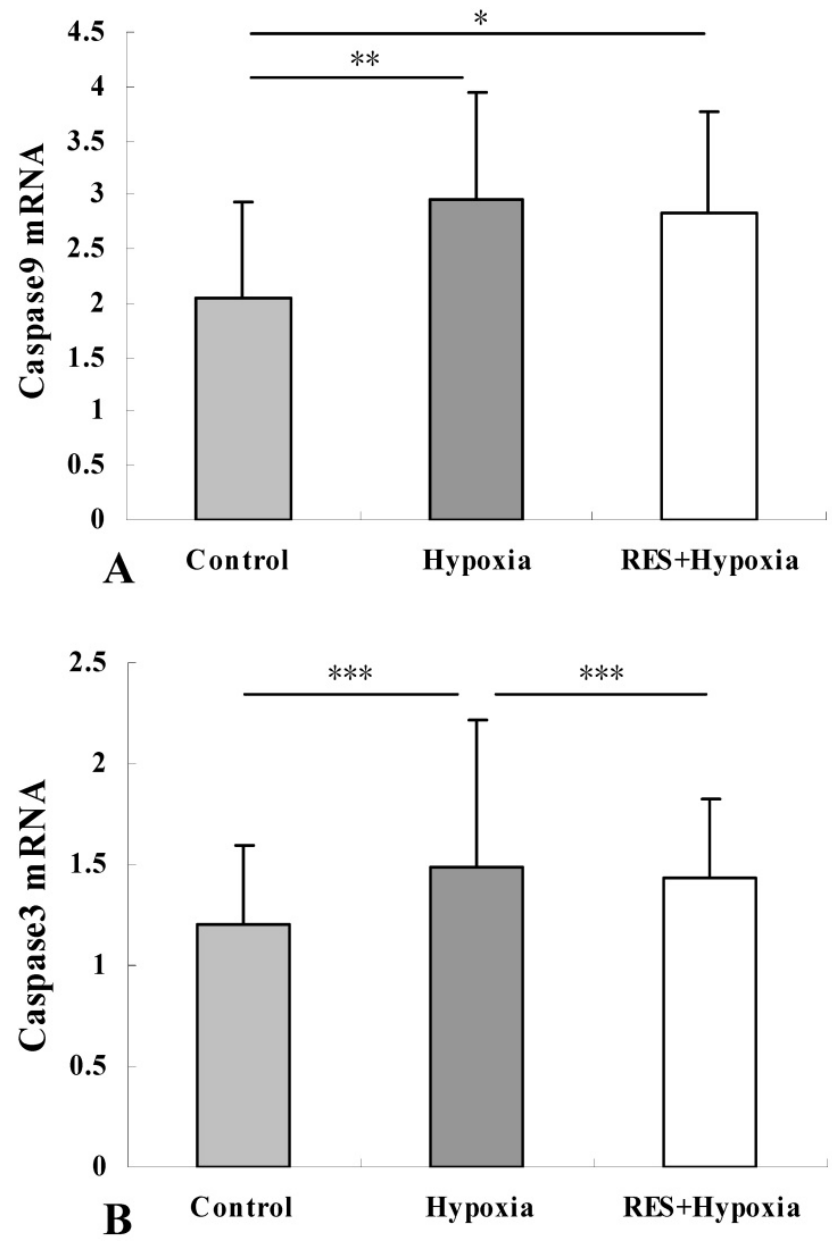

Figure 5. Caspase 9 mRNA (A) and caspase 3 mRNA (B) expression in retinas in control, hypoxia and resveratrol invention (RES + Hypoxia) groups. Results are presented as mean \pm standard deviation (SD) $(n=6)$. All experiments were performed at least in triplicate. $* P<0.05$, $* * P<0.01$ and $* * * P<0.001$.

\section{Discussion}

Traveling to high altitudes is associated with environmental challenges imposed by hypoxia, which contributes to acute altitude illness [1-3]. Hypobaric hypoxia due to the reduced pressure of atmospheric oxygen has several consequences for the oxygen inadequacy of the body [16]. Retina, as the extension 
of central nervous system, is one of the most metabolically active tissues in the human body [17]. Tissue oxygen tension modulates the retinal blood flow. Retina is therefore sensitive to the oxygen alteration and susceptible to oxidative stress because of its high consumption of oxygen $[18,19]$.
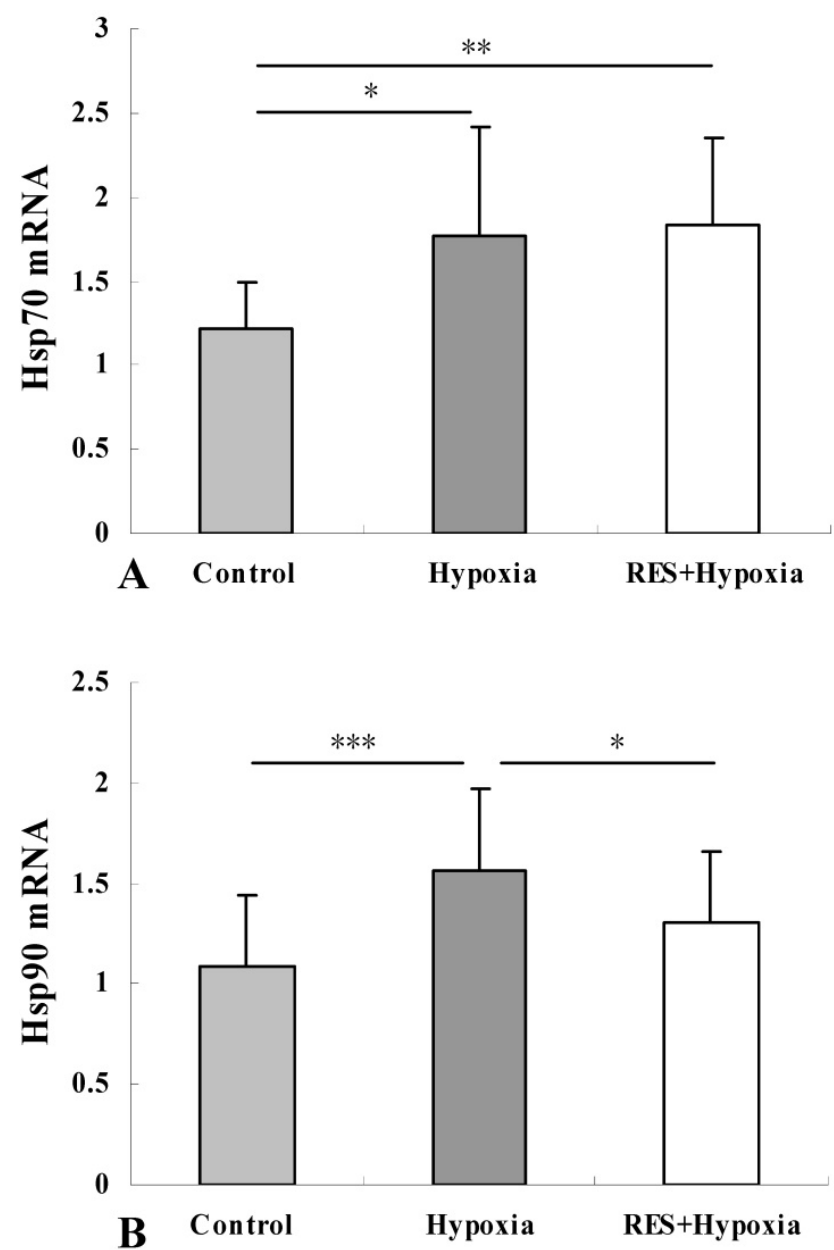

Figure 6. Hsp70 mRNA (A) and Hsp90 mRNA (B) expression in retinas in control, hypoxia and resveratrol invention (RES + Hypoxia) groups. Results are presented as mean \pm standard deviation $(S D)(n=6)$. All experiments were performed at least in triplicate. $* P<0.05, * * P<0.01$ and $* * * P<0.001$.

Hypoxia elicits a cascade of modifications on cellular function and structure [20]. In our study, a significant increase of HIF-1 mRNA level in hypoxia group was observed compared to the controls. HIF-1 is one of the key regulators of hypoxia and is involved in several biological processes, such as angiogenesis, cell proliferation, glucose metabolism, $\mathrm{pH}$ regulation, and apoptosis [21-23]. HIF-1 complex is a transcription factor composed of HIF-1a and HIF-1 $\beta$. HIF-1 is oxidized by hydroxylase enzymes under normoxic condition. However, upon hypoxia, the inhibition of oxygen-dependent degradation of HIF-1a results in accumulated HIF-1a forming a heterodimeric complex with HIF-1 $\beta$, triggering HIF-1 dependent gene expression [21]. Administration of resveratrol in this work attenuated hypoxia-induced upregulation of HIF-1 in the retina, suggesting that resveratrol may have a capacity to counteract the hypoxia stress.

In this study, hypobaric hypoxia increased Txnip, Trx1 and Trx2 expression. Especially, we observed a significant elevation in Trx-1 expression compared to the control group, indicating that redox activity of Trx was triggered under hypobaric hypoxic condition to against the oxidative stress. Txnip, considered as a hypoxia-induced protein, has been reported to be capable of enhancing cellular susceptibility to oxidative stress via binding to $\operatorname{Tr} x$ and inhibiting its disulfide reductase activity [24]. In the hypoxic condition, increased expression of Txnip is postulated to cause the accumulation of reactive oxygen species and apoptotic cell death $[25,26]$. Trx is an intracellular protein secreted under oxidative stress conditions. Trx1 and Trx2 are ubiquitous isoforms of Trx and mainly localized in the cytoplasm and the mitochondria respectively [27]. In the present study, resveratrol treatment significantly suppressed Trx 1 and Trx2 expression in retinas, which might be due to the increased binding of Trx to Txnip, but the detailed functions of Txnip-Trx complex are far from being understood. Besides Trx being regulated by Txnip-Trx complexes, we supposed that the Trx regulation in our study might also be associated with HIF-1 interaction. HIF-1 may be an upstream of Trx expression. Meanwhile, HIF-1 expression itself is controlled by Trx, which promotes biochemical activity of HIF-1 under hypoxic environment, leading to elevated expression of HIF-regulated genes [28]. The interaction between HIF-1 and Trx might be a response to low oxygen tension. Our study provides an indication that resveratrol supplementation might exert anti-oxidative and cytoprotective effect by modulation of Trx1 and Trx2 expression as well as the HIF-1 expression in the retinas to prevent the high altitude-induced retinal disorders.

To investigate the underlying mechanism of the impact of hypoxia on the retina, we examined the possible involvement of apoptosis related genes. Intrinsic and extrinsic pathways are two major pathways for apoptosis induction. Caspase9 and caspase3 operate as the key enzymes in the mitochondria-dependent apoptosis pathway [29- 31]. The hallmarks of the intrinsic pathway are mitochondria involvement and the formation of the apoptosome complex through the released cytochrome c recruiting apoptosis protease-activating factor-1, and this complex consequently cleaves procaspase-9, activates caspase-9 and eventually induces apoptosis via activating the downstream 
caspase-3 [32, 33]. As an indicator of intrinsic mitochondrial pathway of apoptosis, caspase9 showed an increase in mRNA expression under the hypoxic condition in our study. Additionally, elevated caspase 3 mRNA level was also exhibited in retinas in the hypoxia group, further suggesting the activation of the mitochondrial apoptotic pathway. Resveratrol suppressed mRNA expression of the two genes in comparison with untreated group under hypoxic stress, and the effect was more pronounced in caspase3 mRNA expression. These findings demonstrated that deprivation of oxygen induces a pathological impact on the retina by promoting caspase family gene expression and initiating the intrinsic mitochondrial pathway of apoptosis. Resveratrol may have beneficial effects during hypoxia-induced stress by reducing apoptotic signals in retina.

Heat-shock proteins (Hsps), acting as inducible conserved proteins, are activated when exposed to a variety of stress stimuli, such as heat shock, hypoxia, and ischemia [34]. As dominant chaperones, Hsp70 and Hsp90 play crucial roles in protein folding/unfolding, assembly of multiprotein complexes, transport of proteins, cell-cycle control/ signaling, and protecting cells against stress [34, 35]. In the present study, exposure to hypobaric hypoxia resulted in increased Hsp90 expression in retinas, which may be a direct response to cell injuries caused by hypoxia [36]. Hsp90, being capable of binding to HIF-1a, is recognized as a regulator of HIF-1a stability [36,37]. Resveratrol attenuated the hypoxia-induced overexpression of Hsp90 mRNA, further diminished the hypoxia-induced stabilization of HIF-1a and promoted its degradation. Our further analysis on Hsp70 mRNA level revealed that hypoxia evoked Hsp70 mRNA expression, and resveratrol administration further enhanced Hsp70 mRNA level. A large body of evidence has suggested that upregulation of Hsp70 can trigger a cascade of intracellular cytoprotective events and may have a potential to protect cells from stressful injury [38-40]. The results of our study implicated that resveratrol might have a regulatory effect on enhancing the role of Hsp70 in assembly and transport of synthesized proteins within cells, in the removal of denatured proteins [34, 41], and in extenuating acute challenges from internal or external environmental stimuli that pose a threat to normal function of retina. In addition, Hsp70 has been shown to be involved in anti-apoptosis process by blocking recruitment of caspases to the apoptosome complex and inhibiting caspase3-mediated apoptosis [42, 43]. Our results suggest a possibility that the effect of resveratrol on the prevention of high altitude retinal dysfunctions might be associated with its anti-apoptotic role by upregulating Hsp70 along with restraining caspase3 and capase 9 mRNA expressions.

Taken together, our findings indicate that hypobaric hypoxia poses a pathological impact on retinas, and travelers exposed to high altitude and low barometric pressure might therefore be susceptible to impairment of retinal function. Resveratrol exerts its anti-oxidative role by modulating hypoxia stress-associated genes such as HIF-1 and Trx, as well as anti-apoptosis role by regulating apoptosis-related cytokines. Resveratrol might be beneficial to mitigate hypobaric hypoxia-induced retinal dysfunctions, and would be administered as a potential therapeutic way for prophylaxis and treatment of high altitude retinopathy.

\section{Acknowledgements}

This work was supported by Grants from the National Natural Science Foundation of China (81160122 and 81460086), Ministry of Human Resources and Social Security of PRC, China Scholarship Council, Qinghai Science Technology Committee (2014-ZJ911).

\section{Competing Interests}

The authors have declared that no competing interest exists.

\section{References}

1. Schoene RB. Illnesses at high altitude. Chest. 2008; 134: 402-16

2. Clarke C. Acute mountain sickness: medical problems associated with acute and subacute exposure to hypobaric hypoxia. Postgrad Med J. 2006; 82: 748-53

3. Butler FK, Harris DJ, Reynolds RD. Altitude retinopathy on Mount Everest, 1989. Ophthalmology. 1992; 99: 739-46

4. Singh I, Khanna PK, Srivastava MC, Lal M, Roy SB, Subramanyam CS. Acute mountain sickness. N Engl J Med. 1969; 280: 175-84

5. McFadden DM, Houston CM, Sutton JR, Powles AC, Gray GW, Roberts RS. High-altitude retinopathy. JAMA. 1981; 245: 581-86

6. Rennie D, Morrissey J. Retinal changes in Himalayan climbers. Arch Ophthalmol. 1975; 93: 395-40

7. Delmas D, Aires V, Limagne E, Dutartre P, Mazue F, Ghiringhelli F, et al. Transport, stability, and biological activity of resveratrol. Ann NY Acad Sci. 2011; 1215: 48-59

8. Chu LM, Lassaletta AD, Robich MP, Sellke FW. Resveratrol in the prevention and treatment of coronary artery disease. Curr Atheroscle Rep. 2011; 13: 439-46

9. Hamza SM, Dyck JR. Systemic and renal oxidative stress in the pathogenesis of hypertension: modulation of long-term control of arterial blood pressure by resveratrol. Front Physiol. 2014; 5: 292

10. Yavuz S, Aydin NE, Celik O, Yilmaz E, Ozerol E, Tanbek K. Resveratrol successfully treats experimental endometriosis through modulation of oxidative stress and lipid peroxidation. J Cancer Res Ther. 2014; 10: 324-9

11. Andrade JM, Paraíso AF, de Oliveira MV, Martins AM, Neto JF, Guimarães $\mathrm{AL}$, et al. Resveratrol attenuates hepatic steatosis in high-fat fed mice by decreasing lipogenesis and inflammation. Nutrition. 2014; 30: 915-9

12. Trapp V, Parmakhtiar B, Papazian V, Willmott L, Fruehauf JP. Anti-angiogenic effects of resveratrol mediated by decreased VEGF and increased TSP1 expression in melanoma-endothelial cell co-culture. Angiogenesis. 2010; 13: 305-15

13. Albani D, Polito L, Signorini A, Forloni G. Neuroprotective properties of resveratrol in different neurodegenerative disorders. Biofactors. 2010;36: 370-6

14. Burkitt MJ, Duncan J. Effects of trans-resveratrol on copper-dependent hydroxyl-radical formation and DNA damage: evidence for hydroxyl radical scavenging and a novel, glutathione-sparing mechanism of action. Arch Biochem Biophys. 2000; 381: 253-63 
15. Ravagnan L, Gurbuxani S, Susin SA, Maisse C, Daugas E, Zamzami N, et al. Heat-shock protein 70 antagonizes apoptosis-inducing factor. Nat Cell Biol. 2001; 3:839-43

16. Hackett PH, Roach RC. High-altitude illness. N Engl J Med. 2001; 345: 107-14

17. Anderson B Jr, Saltzman HA. Retinal oxygen utilization measured by hyperbaric blackout. Arch Ophthalmol. 1964, 72:792-5

18. Eperon $\mathrm{G}$, Johnson $\mathrm{M}$, David NJ. The effect of arterial $\mathrm{PO}_{2}$ on relative retinal blood flow in monkeys. Investig Ophthalmol. 1975; 14: 342-52

19. Beatty S, Koh H, Phil M, Henson D, Boulton M. The role of oxidative stress in the pathogenesis of age-related macular degeneration. Surv Ophthalmol. 2000; 45: $115-134$

20. Cursio R, Miele C, Filippa N, Van Obberghen E, Gugenheim J. Liver HIF-1 alpha induction precedes apoptosis following normothermic ischemia-reperfusion in rats. Transplant Proc. 2008; 40: 2042-5

21. Semenza GL. Expression of hypoxia-inducible factor 1: mechanisms and consequences. Biochem Pharmacol. 2000; 59: 47-53

22. Hewitson KS, Schofield CJ. The HIF pathway as a therapeutic target. Drug Discov Today. 2004; 9:704-11

23. Koh MY, Spivak-Kroizman TR, Powis G. HIF-1 regulation: not so easy come, easy go. Trends Biochem Sci. 2008; 33: 526-34

24. Patwari P, Higgins LJ, Chutkow WA, Yoshioka J, Lee RT. The interaction of thioredoxin with Txnip: evidence for formation of a mixed disulfide by disulfide exchange. J Biol Chem. 2006; 281: 21884-91

25. Chen J, Saxena G, Mungrue IN, Lusis AJ, Shalev A. Thioredoxin-interacting protein: a critical link between glucose toxicity and beta-cell apoptosis. Diabetes. 2008; 57: 938-44

26. Chen J, Hui ST, Couto FM. Thioredoxin-interacting protein deficiency induces Akt/Bcl-xL signaling and pancreatic beta-cell mass and protects against diabetes. Faseb J. 2008; 22: 3581-94

27. Yoshihara E, Masaki S, Matsuo Y, Chen Z, Tian H, Yodoi J. Thioredoxin/Txnip: redoxisome, as a redox switch for the pathogenesis of diseases. Front Immunol. 2014; 4: 514

28. Zhou J, Damdimopoulos AE, Spyrou G, Brüne B. Thioredoxin 1 and thioredoxin 2 have opposed regulatory functions on hypoxia-inducible factor-1alpha. J Biol Chem. 2007; 282: 7482-90

29. Johnstone RW, Ruefli AA, Lowe SW. Apoptosis: a link between cancer genetics and chemotherapy. Cell. 2002; 108: 153-64

30. Porter AG, Janicke RU. Emerging roles of caspase-3 in apoptosis. Cell Death Differ. 1999; 6: 99-104

31. Kaufmann SH, Lee SH, Meng XW, Loegering DA, Kottke TJ, Henzing AJ, et al. Apoptosis-associated caspase activation assays. Methods. 2008; 44: 262-72

32. Hedskog L, Zhang S, Ankarcrona M. Strategic role for mitochondria in Alzheimer's disease and cancer. Antioxid Redox Signal. 2012; 16:1476-91

33. Rodriguez J, Lazebnik Y. Caspase-9 and APAF-1 form an active holoenzyme. Genes Dev. 1999; 13: 3179-84

34. Hartl FU. Molecular chaperones in protein folding. Nature.1996; 381: 571-9

35. Sõti C, Nagy E, Giricz Z, Vígh L, Csermely P, Ferdinandy P. Heat shock proteins as emerging therapeutic targets. Br J Pharmacol. 2005; 146: 769-80

36. Minet E, Mottet D, Michel G, Roland I, Raes M, Remacle J, et al. Hypoxia-induced activation of HIF-1: role of HIF-1alpha-Hsp90 interaction. FEBS Lett. 1999; 460: 251-6

37. Katschinski DM, Le L, Heinrich D, Wagner KF, Hofer T, Schindler SG, et al. Heat induction of the unphosphorylated form of hypoxiainducible factor-1a is dependent on heat shock protein-90 activity. J Biol Chem. 2002; 277: 9262-7

38. Okubo S, Wildner O, Shah MR, Chelliah JC, Hess ML, Kukreja RC. Gene transfer of heat-shock protein 70 reduces infarct size in vivo after ischemia/reperfusion in the rabbit heart. Circulation. 2001; 103: 877-81

39. Radford NB, Fina M, Benjamin IJ. Moreadith RW, Graves KH, Zhao P, et al. Cardioprotective effects of 70-kDa heat shock protein in transgenic mice. Proc Natl Acad Sci U S A. 1996; 93: 2339-42

40. Rajdev S, Hara K, Kokubo Y, Mestril R, Dillmann W, Weinstein PR, et al. Mice overexpressing rat heat shock protein 70 are protected against cerebral infarction. Ann Neurol. 2000; 47: 782-91

41. Kiang JG, Tsokos GC. Heat shock protein 70 kDa: molecular biology, biochemistry, and physiology. Pharmacol Ther. 1998; 80: 183-201

42. Beere HM, Wolf BB, Cain K, Mosser DD, Mahboubi A, Kuwana T, et al. Heat-shock protein 70 inhibits apoptosis by preventing recruitment of procaspase-9 to the Apaf-1 apoptosome. Nat Cell Biol. 2000; 2: 469-75

43. Lee JS, Lee JJ, Seo JS. Hsp70 deficiency results in activation of c-Jun N-terminal Kinase, extracellular signal-regulated kinase, and caspase-3 in hyperosmolarity-induced apoptosis. J Biol Chem. 2005; 280: 6634-41 